\title{
Inquire for iPad: A Biology Textbook That Answers Questions
}

\author{
Aaron Spaulding ${ }^{1}$, Adam Overholtzer ${ }^{1}$, John Pacheco ${ }^{1}$, Jing Tien ${ }^{1}$, \\ Vinay K. Chaudhri ${ }^{1}$, Dave Gunning ${ }^{2}$, and Peter Clark ${ }^{2}$ \\ ${ }^{1}$ SRI International, 333 Ravenswood Ave, Menlo Park, CA, 94025, USA \\ \{spaulding, overholtzer, pacheco, tien\}@ai.sri.com, \\ vinay.chaudhri@sri.com \\ ${ }^{2}$ Vulcan Inc., 505 Fifth Ave, Suite 900, Seattle WA, 98104, USA \\ $\{$ DaveG, Peterc $\}$ @vulcan.com
}

Textbooks are increasingly moving into the digital realm, which presents an opportunity for them to evolve from providing the reader with a static, linear experience, into an interactive application that can adapt to a student as well as to specific learning goals. As a step in this direction, we present Inquire: Biology, an electronic textbook that provides question-answering capability.

Inquire: Biology, is a novel electronic textbook that runs on an iPad and embeds in it a rich Biology knowledge base and reasoning system. As a student reads the textbook using Inquire, he or she may ask it questions about aspects of the material that are difficult to understand. Inquire can provide answers to these questions as well suggest additional questions based on the student's context.

Inquire is an iPad application consisting of three main components: (1), a Biology textbook, which users can highlight and annotate as desired; (2), the question-asking component, which consists of suggested questions, an option for the user to ask freeform questions, and answers; and (3), a set of glossary pages, which contain text capturing the key points about a concept and interactive concept maps.

The Inquire application connects to a server running our AURA[1] system, which contains a knowledge base (KB) of biology concepts created from a Biology textbook. AURA can interpret questions posed in simplified natural language, and can produce answers and explanations for questions by reasoning over the KB. For a given section of a textbook that a student may be reading, AURA generates questions, which can help a student review the material they have read and explore the sections of the textbooks that they may not have read.

We have conducted an initial round of user studies, and received invaluable input from a number of different domain experts. The prototype indicates that our reasoning and question asking technology can add useful functionality to an electronic textbook. Inquire sets up an inspiring vision towards the textbook of future and can provide a concrete platform in which other educational researchers can plug in their pedagogical approaches and show immediate impact.

Acknowledgement. This work has been funded by Vulcan, Inc.

\section{Reference}

1. Gunning, D., Greaves, M., Chaudhri, V., et al.: Project Halo Update-Progress Towards Digital Aristotle. AI Magazine 31(3) (2010) 